\title{
Estrategias espacio-temporales de las mujeres rurales en el uso de los servicios locales
}

\author{
Rosa Maria López Garcia *
}

\begin{abstract}
RESUMEN
ABSTRACT

Partiendo del hecho de que uno de los

Started from the fact that rural elementos específicos de la sociedad rural es la baja densidad de infraestructuras y servicios, en este estudio se resalta la importancia de conocer tanto la localización de los servicios como la accesibilidad a éstos por parte de los habitantes de zonas rurales, dado que el sistema de infraestructuras tiene un papel fundamental en la comprensión de los modelos territoriales $y$ supone un campo imprescindible para que las politicas de desarrollo rural recuperen

la cantidad y la calidad de los equipamientos como un reto para mantener a la población.

Para el acercamiento al conocimiento del uso, grado de satisfacción y necesidades de infraestructuras por parte de la población rural, se aporta un marco teórico de referencia y una propuesta metodológica, basada en areas are in essence places where activities and services are widely spaced and where there are less services than in urban areas, this article aims to stand out the importance of knowing both the service location and the accessibility to all of them by the rural people. Infrastructures have an important role to understand territorial models and it will be interest to rural development policy for meeting the rural inaccessibility problem and the restructuring of the services in order to fix the population. It is given a theoretical approach and it is proposed a methodology, based on spacetime budgets, to analyse the use, satisfaction and
\end{abstract}

* Universidad Complutense de Madrid. 
las estrategias espacio-temporales de las mujeres rurales, en la medida en que son éstas las principales usuarias de determinados servicios

PALABRAS CLAVE: áreas rurales, servicios, mujer, presupuestos del tiempo. necessities of services by

the rural women, as the

main users of several

services.

KEYWORDS:

rural areas, services, women, spacetime budget.

\section{INTRODUCCIÓN}

Desde finales del siglo pasado las bases teóricas de la geografía social anglosajona destacaban la importancia del espacio y de la localización de los servicios en el bienestar de la sociedad. A pesar de que uno de los elementos que siguen siendo específicos de la sociedad rural es la baja densidad de infraestructuras y servicios, son escasos los estudios sobre el medio rural que incorporan los planteamientos de la geografía del bienestar y aún más escasos los que introducen la variable género. Desde el punto de vista de la investigación consideramos necesario resaltar la importancia de la localización y la accesibilidad a los servicios de los habitantes de zonas rurales, dado que de la caracterización de los equipamientos puede derivarse información complementaria sobre las condiciones de vida y el grado de bienestar de la población. Por otro lado, la identificación de posibles carencias permite no sólo conocer los efectos de las políticas aplicadas a un determinado territorio, sino que también permite detectar necesidades futuras.

Dado que los equipamientos ${ }^{1}$ en el medio rural están muy concentrados espacialmente, el grado de bienestar de la población depende no sólo de la distancia sino también de las condiciones socioeconómicas del desplazamiento, por lo que resulta necesario identificar las respuestas diferenciadas de los habitantes en el uso de los servicios, resultándonos especialmente interesante el comportamiento de las mujeres. La responsabilidad del trabajo reproductivo (cuidado de niños y ancianos, por ejemplo), asumido por las mujeres mayoritariamente, implica que son éstas las principales usuarias de determinados servicios, por lo que las deficiencias en éstos afectan más a las mujeres que a los hombres (ausencia de guarderías, lejanía de servicios sanitarios, etc.).

1 Entendemos por equipamientos «el conjunto de los edificios y de las instalaciones que permiten asegurar a la población los servicios colectivos de los que tiene necesidad", detalladamente analizados en MOPU (1982): Op.cit. 
El objetivo del presente estudio es resaltar la importancia de conocer el entorno cotidiano donde las mujeres rurales llevan a cabo tanto el trabajo reproductivo como el productivo, el uso que hacen de los servicios locales y de los recursos de los que disponen para desplazarse. A través de una metodología cualitativa, basada en la entrevista en profundidad y en los presupuestos del tiempo, es posible conocer el uso y el grado de satisfacción que las mujeres manifiestan tener respecto a las dotaciones existentes en su entorno y de este modo colaborar a que las políticas de desarrollo rural recuperen la cantidad y la calidad de los servicios como un reto imprescindible para mantener a la población.

\section{LA GEOGRAFIA DEL BIENESTAR}

Dado que el medio rural ocupa la mayor parte del territorio, tanto a nivel europeo como nacional, y a pesar de que se trate de un territorio dependiente del urbano, no se puede obviar el importante papel que desempeña no sólo desde el punto de vista de la vertebración territorial, sino también desde una perspectiva medioambiental. La riqueza del patrimonio rural, el impulso de las iniciativas de desarrollo local y la multifuncionalidad de estos espacios los convierten en medios que exigen una lectura dinámica, en constante revisión. Esta revisión pasa por considerar que el sistema de equipamientos e infraestructuras tienen un papel fundamental a la hora de analizar un determinado modelo territorial. En ese sentido, los geográfos y demás científicos sociales debemos plantearnos algunos interrogantes sobre el devenir de los espacios rurales, siendo una de sus problemáticas específicas la necesidad de reforzar las dotaciones de infraestructuras y equipamientos, dado que los territorios rurales sufren aislamiento, desequilibrios territoriales y dependencia de las áreas metropolitanas.

La baja densidad de infraestructuras y servicios es uno de los elementos que siguen siendo específicos de la sociedad rural, sin embargo, apenas existen investigaciones de espacios rurales realizados desde los planteamientos teóricos de la geografía del bienestar. En general estas tendencias han centrado su atención en el espacio urbano, donde los contrastes socioeconómicos son más evidentes.

Dentro del grupo de geógrafos anglosajones que a finales de los años setenta comenzaron a plantear la necesidad de incorporar a la geografía la óptica del compromiso y de las preocupaciones sociales, podemos afirmar que el concepto de bienestar en geografía fue incorporado por D.M. Smith, quien en su reconocida obra Geografía Humana (1980) abordaba la 
geografía humana como un análisis del bienestar social en el espacio. Para este autor, el objeto principal de la geografía humana debería ser los seres humanos, por lo que «el interés principal se centrará entonces en la cualidad de sus vidas. Las características demográficas, la actividad económica, la organización del espacio, la difusión de innovaciones, etc (...) contribuyen a la diferenciación del territorio en relación al bienestar de quienes lo habitan" (Smith, 1980: 543). Aunque aplicadas al medio urbano, las bases teóricas de Smith, que considera el espacio como elemento clave en la localización de los servicios y, por lo tanto, en el bienestar de la población, ofrece unos objetivos de singular valor para ser aplicados al medio rural.

El acercamiento del geógrafo rural al tema del bienestar supone dar respuesta a interrogantes tales como en qué medida el espacio condiciona la calidad de vida (en función de la distancia, el acceso a los servicios y las diferencias sociales), o en qué medida las diferencias territoriales inciden en los niveles de bienestar, abordando tanto los desequilibrios entre medio rural y urbano, y las desigualdades existentes dentro del propio medio rural.

Los primeros en incorporar el tema del bienestar a las zonas rurales han sido los geógrafos británicos, destacando, entre otros, Shaw (1979), Pacione (1983) y Clark y Lockhart (1984). En la geografía rural española destacamos a Sabaté, Piris y Salvador (1985) y Sabaté (1987) como autores que han incorporado los temas de bienestar y cuyos estudios ponen de manifiesto las relaciones entre estructura de la población, la evolución demográfica y el deterioro de los servicios.

\section{EL BIENESTAR EN ZONAS RURALES}

Uno de los elementos específicos de la sociedad rural es la baja densidad de servicios, si bien es cierto que se dan diferentes situaciones según se trate de zonas marginales o zonas evolucionadas, situadas éstas últimas próximas a áreas urbanas o ejes de expansión. Por otro lado, la política de localización de servicios públicos ha seguido el camino de la lógica de la rentabilidad económica, lo que ha supuesto la desaparición de escuelas, transportes públicos, etc., de pequeños núcleos rurales. En efecto, el despoblamiento rural ha conllevado una reestructuración de los servicios básicos. La iniciativa privada, ante una población escasa y envejecida, ha disminuido sus actividades, mientras que los servicios públicos, o bien han optado por reducir los servicios prestados o han deteriorado su calidad. Ciertamente algunos servicios y equipamientos pueden tener un carácter puramente local y estar presente prácticamente en todos los mu- 
nicipios, como por ejemplo una escuela primaria, pero otros son característicos de núcleos rurales de una cierta entidad, como los institutos de secundaria. Como hecho que agrava la situación, debemos añadir la realidad que viven aquellas zonas rurales con un intenso grado de envejecimiento, que obliga a considerar el tema del bienestar desde la óptica de una sociedad protagonizada por ancianos, con escasos niveles de renta y de movilidad.

El breve panorama descrito plasma una realidad de contrastes en los niveles de bienestar de la población rural. Estos contrastes pueden ser contemplados desde dos perspectivas: territorial y social. La primera alude a las diferencias territoriales que suponen tanto la localización relativa de los núcleos de población (topografía, vias de comunicación, distancia a núcleos urbanos) como la estructura del poblamiento (tamaño de los núcleos de población). Por otro lado, las diferencias individuales o sociales inciden en el hecho de que hay grupos sociales con menores posibilidades de accesibilidad a los servicios, en función de la edad, género y/o rentas.

Desde el punto de vista de la investigación consideramos necesario resaltar la importancia de la caracterización de los equipamientos, la localización y la accesibilidad a los mismos por parte de los habitantes de zonas rurales, dado que de este análisis puede derivarse información complementaria sobre las condiciones de vida y el grado de bienestar de la población. Por otro lado, la identificación de posibles carencias permite no sólo conocer los efectos de las políticas aplicadas a un determinado territorio, sino que también permite detectar necesidades futuras, en la medida en que las políticas de desarrollo en zonas rurales insisten en la necesidad de recuperar, en cantidad y calidad, los servicios como uno de los retos que ayuden a mantener, e incluso atraer, a la población rural.

La concepción de nuevos espacios de desarrollo exige una lectura e interpretación del territorio donde se supere la concepción de éste como mero soporte de actividades económicas y sea entendido como un recurso de dimensiones múltiples, dado que todos los territorios disponen de un conjunto de recursos que constituyen su potencial de desarrollo. Desde la Unión Europea se viene debatiendo una nueva definición de la ordenación del territorio con el fin de abordar estrategias de desarrollo territorial que superen, por un lado, los problemas de la congestión urbana y, por otro lado, los de despoblación y abandono de zonas rurales (ETE, 1999). Desde la Estrategia Territorial Europea (ETE) se plantea la necesidad de un desarrollo territorial policéntrico que fomente el equilibrio territorial, para lo que se recomienda la promoción de estrategias que apoyen la complementariedad de los medios urbano y rural. 
Para la Unión Europea una de las claves del desarrollo policéntrico está en que todas las regiones tengan un acceso adecuado a las infraestructuras, con el fin de fomentar la cohesión económica, social y territorial. Para la ETE no es posible reducir las disparidades territoriales de la Unión Europea sin la mejora de los servicios e infraestructuras de transporte, por lo que resalta la estrecha relación entre transporte, infraestructuras y servicios y un desarrollo asociado, integrado y policéntrico.

En esta nueva meta de la política europea, la funcionalidad de los espacios es clave, siendo las pequeñas ciudades elementos esenciales en las relaciones entre el mundo rural y el urbano. Al situarse próximas a los núcleos rurales, las pequeñas ciudades constituyen un elemento fundamental en la mejora del bienestar y la calidad de vida de la población y el equilibrio territorial. Las pequeñas ciudades ofrecen servicios, tanto públicos (sanidad, educación), como privados (comercio, financieros) que contribuyen a dinamizar el medio rural. Por otro lado, las pequeñas ciudades concentran gran parte del empleo no agrario de sus entornos, por lo que contribuyen a diversificar la economía comarcal, atraer nuevas actividades y mejorar el mercado de trabajo. Las oportunidades de empleo que ofrecen las pequeñas ciudades son muchas veces aprovechadas por los habitantes rurales de los municipios próximos, quienes realizan desplazamientos pendulares, que son posibles gracias tanto a la mejora de la red de carreteras como al aumento de la motorización privada. Sin embargo, no todos los habitantes rurales tienen las mismas posibilidades de elección, ya que un número importante, en función de la edad, género o rentas, no dispone de automóvil y necesita del transporte público, siendo éste, por lo tanto un servicio realmente importante para la dinamización del mercado de trabajo en comarcas rurales y la fijación de población en sus núcleos de residencia. De hecho, en aquellas áreas de baja densidad de población son numerosos los municipios que carecen de transporte público y se asiste a soluciones alternativas, como los taxis colectivos.

\section{ELEMENTOS DE BIENESTAR EN ZONAS RURALES}

Es ampliamente reconocida la necesidad de realizar lecturas dinámicas del actual medio rural, muy diversificado y con nuevas funcionalidades, impulsadas por las políticas públicas de la Unión Europea, las iniciativas de desarrollo local, etc. Tal y como se refleja en el trabajo Análisis de variables y representación cartográfica para la caracterización y clasificación del medio rural español (MOPTMA, 1995), es necesario trabajar con una gran variedad de indicadores que nos permitan establecer una tipología de 
espacios rurales. El citado trabajo, utilizando el municipio como unidad de análisis, agrupa los indicadores en cinco categorías: condicionantes físicoterritoriales, estructura demográfica, estructura ocupacional, aspecto de la ruralidad y caracterización socioeconómica. Dentro de las dos últimas categorías, el trabajo incluye todos aquellos indicadores relativos a niveles y tipologías de servicios, tipologías de viviendas, infraestructuras en telecomunicaciones, etc. De este trabajo se desprende la idea sobre la que venimos insistiendo a lo largo del presente estudio: el sistema de infraestructuras tiene un papel fundamental en la comprensión de un modelo territorial y supone un campo imprescindible de la acción territorial.

Para crear nuevos espacios de desarrollo es necesario, en primer lugar, realizar una lectura actualizada del medio rural donde se diferencien los niveles y las tipologías de ruralidad. Una visión territorial donde se plasmen las problemáticas del poblamiento, las problemáticas funcionales y las necesidades de la población en el uso de bienes y servicios. Consideramos que la calidad de vida de los habitantes es uno de los grandes retos del desarrollo territorial sostenible.

Para conseguir el objetivo señalado resulta imprescindible elaborar indicadores significativos de calidad de vida aplicados al medio rural, considerando cuáles son los aspectos de mayor interés para la sociedad rural. Recuperando los indicadores de bienestar aplicados en diferentes trabajos (Sabaté, Piris y Salvador, 1985; Sabaté, 1987) éstos se agrupan en varias categorías:

- Condiciones de la vivienda, equipamientos y dotaciones básicas del hogar (electricidad, agua caliente, calefacción,...).

- Infraestructuras: servicios colectivos, como abastecimiento de agua, evacuación, depuradoras, recogida de basuras, iluminación viaria, pavimentación,...

- Servicios básicos: comercio, sanitarios en diferentes niveles (farmacia, ambulatorio...), docentes en distintas categorías (primaria, secundaria...), bibliotecas, instalaciones deportivas...

- Movilidad: número de automóviles privados, servicio de transportes colectivos...

Los elementos que más influyen en la calidad de vida de la población rural son bastante diferentes a los que existen en las ciudades. En encuestas llevadas a cabo en algunos trabajos, los habitantes rurales destacan la importancia de las condiciones materiales en la vivienda y en el pueblo (pavimentación de las calles, teléfono, transportes...) para sus 
vidas cotidianas. Sin embargo, el problema que en mayor medida se detecta es el del aislamiento: la insuficiencia de servicios básicos y su alejamiento y el mal o inexistente funcionamiento de los transportes públicos dificultan hechos tan básicos como realizar compras o acudir a un médico especialista (Sabaté, 1989).

Es evidente que dentro del medio rural existen grandes diferencias de calidad de vida en función del número y la calidad de los indicadores arriba mencionados. Las decisiones políticas de localización de servicios juega, en ese sentido, un papel fundamental, así como la accesibilidad y las infraestructuras de transporte. Dado que los servicios están muy concentrados espacialmente en el medio rural (hospitales, institutos de secundaria, comercios,...), la población depende de la distancia y las posibilidades socioeconómicas de desplazamiento.

La accesibilidad es uno de los temas más tratados como medida del bienestar de la población en zonas rurales, si bien es cierto que destacan los análisis centrados en áreas urbanas. Pionero es el trabajo de M.J. Moseley Accesibility: the rural change (1979), quien analiza lo que considera el principal problema del medio rural británico: la inaccesibilidad a la que se ven sometidos los habitantes rurales, dada la escasez de transporte público y la distancia, muchas veces grande, entre núcleos y servicios. Estimamos que las mayores aportaciones que lleva a cabo Moseley son, en primer lugar, el considerar que en los problemas de accesibilidad en las zonas rurales hay condicionamientos socioeconómicos, dado que son los pobres, los niños y las mujeres quienes sufren en mayor medida el alejamiento de los servicios públicos; por otro lado, el citado autor considera el concepto de accesibilidad como una medida de éxito o fracaso de las políticas públicas.

En la geografía española resultan innovadores los trabajos que lleva a cabo Gutiérrez Puebla (1988 a, b), desde una perspectiva social, sobre la accesibilidad y el transporte en zonas rurales. Dentro de los trabajos que se han realizado sobre el tema en la actualidad destacamos el de Gray et al. (2001) sobre la dependencia del coche y el transporte público en la Escocia rural.

\section{LAS MUJERES RURALES Y EL USO DE LOS SERVICIOS}

Dado que los servicios están muy concentrados espacialmente en el medio rural, el grado de bienestar de la población depende no sólo de la distancia sino también de las condiciones socioeconómicas del desplaza- 
miento, por lo que resulta necesario identificar las respuestas diferenciadas de los habitantes en el uso de los servicios, resultándonos especialmente interesante el comportamiento de las mujeres.

Resulta esencial para caracterizar e identificar las posibles carencias o necesidades de equipamientos e infraestructuras en zonas rurales, partir del hecho de que las peculiares relaciones de género definen una sociedad rural sometida a profundos cambios. Las áreas rurales son mucho más complejas y dependientes de los esquemas de funcionamiento de las aglomeraciones urbanas y precisan nuevos enfoques. Desde nuestro punto de vista, las condiciones personales de movilidad, la localización de los equipamientos y las diferentes funciones según género, conllevan un acceso diferente a los servicios y, por lo tanto, a distintos niveles de bienestar. Para Sabaté (1989) la perspectiva de género debe incidir en dos aspectos referidos a las diferencias según género en cuanto a la utilización de los servicios, por un lado, y los sistemas de transporte empleados por hombres y mujeres, por otro lado.

Son escasos los trabajos que hayan centrado su análisis en las condiciones de vida y el acceso a los servicios en relación a su incidencia sobre las mujeres. Innovador en este sentido resulta la nbra de Sabaté (1989) Las mujeres en el medio rural, donde dedica un capítulo a las condiciones de vida, ocio y relaciones sociales, en concreto a la localización $y$ acceso a los servicios por parte de las mujeres, incluido el tema de los transportes y la accesibilidad. Más recientes son los trabajos de Vera y Rivera (1999) y Prados Velasco (2000); los primeros dedican un apartado a las condiciones de habitabilidad de los pueblos, analizando los tipos de vivienda y los equipamientos del hogar. Prados Velasco, en un capítulo dedicado a la medida del bienestar social y condiciones de vida de la población también se centra en las características de las viviendas y los equipamientos básicos.

En relación a la disponibilidad de equipamientos de ocio en zonas rurales, Silvestre y Aristegui (2000) analizan el tema desde una perspectiva de género y mediante la aplicación de técnicas tanto cuantitativas (encuestas para detectar diferentes percepciones) como cualitativas (entrevistas en profundidad). Las citadas autoras, que llevan a cabo su estudio en el medio rural vasco, concluyen que no se ofrecen suficientes alternativas a las mujeres para disfrutar de su tiempo libre.

El tema de la movilidad y accesibilidad en áreas rurales desde el punto de vista del género ha recibido, sin embargo, un escaso tratamiento. La mayoría de los estudios se centran en áreas urbanas (Rosebloom, 1989). También es cierto que los problemas de accesibilidad-movilidad comienzan a 
destacar dentro de la bibliografía sobre mujeres en áreas rurales, si bien es cierto que insertados en temáticas más amplias (Villarino y Armas, 2000).

Desde nuestro punto de vista resulta imprescindible acercarse al conocimiento del uso, grado de satisfacción y necesidades de servicios e infraestructuras por parte de la población rural desde una perspectiva de género. En la medida en que las mujeres asumen mayoritariamente la responsabilidad del trabajo reproductivo (cuidado de niños y ancianos, por ejemplo), éstas son las principales usuarias de determinados servicios, por lo que las deficiencias en éstos afectan más a las mujeres que a los hombres (ausencia de guarderías, lejanía de servicios sanitarios, etc.). En la medida en que conozcamos el entorno cotidiano donde las mujeres rurales llevan a cabo tanto el trabajo reproductivo como el productivo, el uso que hacen de los servicios locales y de los recursos de los que disponen para desplazarse podremos ayudar a que las políticas de desarrollo rural recuperen la cantidad y la calidad de los servicios como un reto imprescindible para mantener a la población.

El principal interrogante que surge ante la necesidad de incorporar la perspectiva de género en el análisis de servicios e infraestructuras en áreas rurales es ¿en qué medida incide la localización y el acceso a los servicios en las mujeres rurales? Para poder dar respuesta a esta cuestión es necesario conocer el modo y la frecuencia con que éstas hacen uso de los servicios locales.

Las mujeres rurales desarrollan numerosas actividades: son educadoras de sus hijos, agricultoras, dinamizadoras sociales, cuidadoras de la familia, etc. En este sentido, las mujeres son las principales usuarias de los servicios, son ellas, por ejemplo, las que llevan a los niños a la escuela, realizan las compras, acompañan a los ancianos de la familia al médico. Es evidente que, de este modo, les afecta muy directamente tanto la calidad y cantidad de servicios, como el acceso a este tipo de centros.

En relación a los servicios educativos y escolares, destacan la prácticamente inexistente presencia de guarderías, lo que limita las posibilidades de movilidad de las madres o el acceso de éstas a un trabajo fuera de la vivienda, constituyéndose el trabajo a domicilio como una alternativa laboral para la mujeres rurales. En relación a este tipo de trabajo Baylina (2000:230) señala que el predominio de las mujeres en este sector «se debe a una combinación entre la pobreza, la falta de alternativas laborales y la responsabilidad sobre el cuidado de los hijos/as y otras personas dependientes, la falta de servicios de atención a la infancia, etc.».

Respecto a los servicios sanitarios, en general podemos afirmar que es la mujer la que tiene asignada la función de ser ella quien vele por la salud 
de su familia, quien lleva a los hijos al médico y acompaña a los ancianos o personas dependientes de la unidad familiar. Es por esto que los servicios sanitarios constituyen otro ejemplo de las deficiencias que puedan derivarse de su localización, teniendo en cuenta que la tendencia ha sido la de concentrar éstos en un reducido número de pueblos.

En relación a los servicios comerciales, la mujer suele ser la principal usuaria del comercio básico en tanto en cuanto que es ésta la que asume del tradicional rol familiar de velar por la subsistencia y alimentación familiar. Por lo tanto, la deficiencia en este servicio recae directamente sobre las mujeres, sobre todo allí donde la baja densidad de población ha supuesto la pérdida de comercios. Siendo el comercio un servicio privado, es evidente que su supervivencia está ligada a la rentabilidad económica del mismo.

El tema de infraestructuras en transportes, movilidad y accesibilidad también incide en las diferencias existentes dentro del medio rural y entre los individuos, según sus posibilidades de movilidad. El transporte público rural es, en general, deficiente en cuanto a escasez, tanto en número como en horarios, y de baja calidad. De este hecho se deriva que sea el transporte en vehículo privado el que solucione los problemas de accesibilidad en la mayoría de los casos. Teniendo en cuenta que la mayoría de la población rural es mayor, es evidente que el factor edad acentúa las dificultades de movilidad. También en este tema se producen, por otro lado, las diferencias entre hombres y mujeres. Si bien es cierto que en general se ha observado un considerable incremento de la movilidad de las mujeres en áreas rurales, hemos de tener en cuenta las grandes diferencias existentes entre las áreas rurales de la geografía española, donde las actitudes y las mentalidades sociales influyen sobremanera. Sabaté (2000:4), refiriéndose a mujeres en zonas rurales de las provincias de Cáceres y Toledo, señala cómo "en 1999 la mayoría tenía carnet de conducir y utilizaba el coche tanto para ir a trabajar como para hacer las distintas gestiones". Totalmente diferente es la situación descrita por Villarino y Armas, quienes analizan el trabajo a domicilio en el sector de la confección en aldeas gallegas, y señalan como el marido, además de su propio trabajo, "se ocupa de lo relacionado con ciertos asuntos bancarios y, por supuesto del coche (es obvia la relación entre movilidad, independencia y tránsito por espacios públicos, de la cual sólo se beneficia él)». En otro párrafo, referido a la economía familiar, las mujeres entrevistadas declaran que «la gasolina es un gasto del marido, porque es quien anda con el coche» (Villarino y Armas, 2000:258-260).

La movilidad deficiente tiene, asimismo, "muchas más consecuencias que el mejor o peor acceso a los servicios. La posibilidad de moverse fa- 
cilita, por ejemplo, el poder acceder a un empleo que esté situado en un lugar distinto al de la residencia" (Sabaté, 1989:150) o el acceso a cursos o estudios. En definitiva, la movilidad espacial facilita el acceso a servicios, trabajos y oportunidades ofrecidas por la sociedad, lo que ayuda a mejorar las situaciones personales dentro de esa sociedad.

\section{LAS ESTRATEGIAS ESPACIO-TEMPORALES DE LAS MUJERES EN EL USO DE LOS SERVICIOS}

Para avanzar en el refuerzo de las dotaciones de equipamientos y servicios en función de las necesidades de la población, además de conocer las disponibilidades existentes en cada núcleo rural, es necesario detectar cuáles son los aspectos de interés preferente para los habitantes, en este caso las mujeres. Se trata de analizar el bienestar de las personas en términos de su espacio vital, tanto en lo referente a las condiciones objetivas (presencia de determinados servicios) como a las valoraciones, que forman parte del bienestar subjetivo. En la medida en que la calidad de vida de los habitantes mejora con la presencia de determinados servicios e infraestructuras, es conveniente conocer cuáles son los elementos que más influyen en sus vidas (condiciones materiales de las viviendas, transportes, servicios sanitarios, ....).

Consideramos que a través de una metodología cualitativa, basada en la entrevista en profundidad, es posible conocer el uso y el grado de satisfacción que las mujeres manifiestan tener respecto a las dotaciones existentes en su entorno y de este modo colaborar al refuerzo de las dotaciones en infraestructuras. Creemos en la conveniencia de aplicar una metodología cualitativa dado que no existen ni datos publicados ni estadísticas que ofrezcan datos significativos sobre el grado de satisfacción y necesidades planteadas por los habitantes rurales. Así pues, el trabajo de campo es imprescindible para llevar a cabo este objetivo y muy en particular la entrevista en profundidad.

Dado que una de las características más singulares de las mujeres rurales es la de simultanear en el tiempo y en el espacio tareas (cuidar ancianos, trabajar en la explotación agrícola y/o en el taller de confección, realizar compras, etc.) y teniendo en cuenta que el tiempo es un bien escaso, resulta de gran interés conocer las estrategias de gestión del tiempo y del espacio por parte de los habitantes rurales. Con la metodología de la Geografía del Tiempo (Díaz Muñoz, 1992) es posible realizar diarios o presupuestos del tiempo, donde se describan y cuantifiquen las actividades realizadas por las mujeres y observar de este modo las posibili- 
dades de movilidad que poseen en la realización de sus tareas y las carencias de servicios que padecen.

En una reciente investigación llevada a cabo en las provincias de Toledo y Cáceres, sobre las estrategias de uso del tiempo y del espacio por mujeres trabajadoras rurales, se constata cómo las mujeres rentabilizan su escaso tiempo minimizando los desplazamientos. Los autores demuestran que la proximidad entre los lugares utilizados en la actividad cotidiana de las mujeres, incluso la proximidad espacial de los servicios, facilita "de forma decisiva la pluriactividad desarrollada por las mujeres así como la superposición del trabajo productivo y reproductivo". Asimismo, se destaca la importancia que tiene el reducido tiempo empleado en los desplazamientos (por debajo de una hora diaria en todos los casos), (...), lo que refuerza la hipótesis de que la proximidad entre los espacios utilizados por las mujeres en zonas rurales se traduce en un aumento de los tiempos dedicados al trabajo" (Sabaté, 2000: 5).

La realización de entrevistas en profundidad permite conocer el grado de satisfacción que las mujeres manifiestan tener respecto a los espacios de residencia y vivienda y de los servicios, dotaciones y equipamientos existentes en torno a sus lugares cotidianos. Además, ccn la realización de entrevistas en profundidad se analizan las estrategias seguidas por las mujeres para afrontar la doble jornada, es decir, el trabajo productivo (explotación agraria, taller de confección, etc.) más reproductivo (cuidado de personas dependientes, tareas del hogar, etc.). Las estrategias que las mujeres siguen respecto a la gestión del tiempo y del espacio, en cualquier caso, están determinadas por el uso y la presencia o ausencia de determinados servicios.

Por otro lado, la utilización de los presupuestos diarios del tiempo permiten recoger una información de carácter cuantitativo que complementen la información, de un carácter más subjetivo, de la entrevista en profundidad.

Con el diario del tiempo se recoge de manera sistemática el uso del tiempo, con la descripción detallada de las tareas desarrolladas, que realiza una persona en un periodo de tiempo determinado. La información combinada de actividad en el tiempo y en el espacio se obtiene al detallar la actividad realizada, el lugar donde se lleva a cabo y la hora del comienzo y final de la misma.

El mayor interés que tienen los presupuestos diarios del tiempo en este caso es que nos informan de las actividades realizadas por las mujeres, poniendo de manifiesto el uso que realmente hacen de los servicios locales. De manera cuantificable resulta conveniente comprobar hasta qué punto son las mujeres las que con mayor frecuencia utilizan los ser- 
vicios y equipamientos de que disponen (realizar las compras, llevar a los ancianos al consultorio médico,...) y si la proximidad o lejanía de éstos convierte en mucho más complicadas sus tareas o, por el contrario, la cantidad y la calidad de infraestructuras son favorables y aligeran sus quehaceres habituales.

Finalmente, destacar que las entrevistas en profundidad nos permiten conocer el grado de satisfacción que las mujeres manifiestan tener respecto a los servicios locales y las estrategias espacio-temporales que desarrollan para ejercer su jornada laboral. Consideramos que es tan importante poder cuantificar y analizar la tipologia de transportes públicos o de comercios especializados de los que dispone un municipio, como tener en cuenta las opiniones manifestadas por los usuarios al respecto.

\section{CONCLUSIONES}

En la medida en que las politicas de desarrollo en zonas rurales insisten en la necesidad de recuperar, en cantidad y calidad, los servicios como uno de los retos que ayuden a mantener, e incluso atraer, a la población rural, consideramos de gran importancia acercarnos desde la geografía a los estudios de los equipamientos e infraestructuras. Por otro lado, las diferencias individuales o sociales inciden en el hecho de que hay grupos sociales con menores posibilidades de accesibilidad a los servicios, en función de la edad, género y/ o rentas, por lo que se dan distintos niveles de bienestar. Desde nuestro punto de vista resulta imprescindible acercarse al conocimiento del uso, grado de satisfacción y necesidades de servicios e infraestructuras por parte de la población rural desde una perspectiva de género.

En la medida en que las mujeres asumen mayoritariamente la responsabilidad del trabajo reproductivo (cuidado de niños y ancianos, por ejemplo), éstas son las principales usuarias de determinados servicios, por lo que las deficiencias en éstos afectan más a las mujeres que a los hombres (ausencia de guarderías, lejanía de servicios sanitarios, etc.) Consideramos que a través de una metodología cualitativa, basada en la entrevista en profundidad, es posible conocer el uso y el grado de satisfacción que las mujeres manifiestan tener respecto a las dotaciones existentes en su entorno y de este modo colaborar al refuerzo de las dotaciones en infraestructuras. Por orto lado, teniendo en cuenta que una de las características más singulares de las mujeres rurales es la de simultanear en el tiempo y en el espacio tareas (cuidar ancianos, trabajar en la explotación agrícola y/o en el taller de confección, realizar compras, etc.) y teniendo en cuenta que el tiempo es un bien escaso, resulta de gran interés conocer 
las estrategias de gestión del tiempo y del espacio por parte de los habitantes rurales. Consideramos que puede ser de gran utilidad realizar diarios o presupuestos del tiempo, donde se describan y cuantifiquen las actividades desarrolladas por las mujeres y observar de este modo las posibilidades de movilidad que poseen en la realización de sus tareas y las carencias de servicios que padecen.

\section{BIBLIOGRAFIA}

Baylina, M. (2000): “Unidas por el mismo hilo: Un estado de la cuestión sobre el trabajo a domicilio", en García Ramón, M. D. y Baylina Ferré, M.(eds.) (2000): Op. cit., (págs. 219 244).

CLARK, G. y LOCKHART, D. (1984): “Deprivation and welfare in rural areas". Área, 16, 2.

COMISIÓN EUROPEA (1999): Estrategia Territorial Europea (ETE). Hacia un desarrollo equilibrado y sostenible de la Unión Europea.

Diaz Muñoz, M. A. (1992): “Espacio y tiempo en la actividad cotidiana de la población", en Bos. QUE, J ; CAstro, C.; Diaz, M. A. y Escobar, F. J., Prácticas de Geografía de la percepción y de la actividad cotidiana. Barcelona, Oikos-tau., págs. 15-44.

Garcia Ramon, M. D. y Baylina Ferré, M. (eds.) (2000). El nuevo papel de las mujeres en el desarrollo rural. Barcelona: Oikos-tau.

Gray, D.; Farrington, J.; Shaw, J.; Martin, S. y Roberts, D. (2001): "Car dependence in rural scotland: Transport policy, devolution and the impact of the fuel duty escalator", Journal of Rural Studies, 17, págs. 113-125

Gutekraez Puebla, J. (1988 a): “Accesibilidad y transporte rural: una perspectiva social». Transporte, Turismo y Comunicaciones, 34, págs. 27-40.

-- (1988 b): “Crisis y perspectivas de futuro en el transporte colectivo del medio rural». Estudios Geográficos, 193, págs. 559-580.

MOPTMA (1995): Análisis de variables y representación cartográfica para la caracterización y clasificación del medio rural español. Madrid, MOPTMA.

MOPU (1982): Estudio comparado de estandares de equipamiento. Madrid, MOPU.

Moseley, M. J. (1979): Accessibility: the rural challenge. London, Methuen \& Co Ltd.

PACIONE (ed.) (1983): Progress in rural Geography. London, Croom Helm.

Prados Velasco, M. J. (2000): Situación socioeconómica de las mujeres rurales en España. Junta de Andalucia. Consejería de Agricultura y Pesca. Monografías 24/00.

ROSENBLOOM, S. (1989): “Differences by sex in the home-to-work travel patterns of married parents in two major metropolitan areas", Espace, Populations, Societes, 1, págs. 65-76.

SABAté, A. (1987): "Geografía social y renovación conceptual en el análisis del medio rural". Anales de Geografia de la Universidad Complutense, 7, págs. 77-84.

- (1989): Las mujeres en el medio rural. Madrid, Ministerio de Asuntos Sociales, Instituto de la Mujer.

- (2000): "Estrategias de uso del tiempo y del espacio por mujeres trabajadoras en zonas rurales", Actas del X Coloquio de Geografía Rural de España, Lleida, septiembre, 2000.

- PIRIS, C. y Salvador, J. (1985): Implicaciones territoriales del cambio demográfico en el medio rural (provincia de Zaragoza). Zaragoza, CEOTMA.

SHAw, J. M. (1979): Rural deprivation and planning. Norwich, Geo Abstract.

Silvestre, M. y Aristegui, I. (2000): "La mujer rural vasca y el ocio. Necesidades, demandas y carencias». Sexto Congreso Mundial de Ocio Ocio y Desarrollo Humano. Bilbao, págs. 3-7, julio, 2000.

SMITH, D. M. (1980): Geografía Humana. Barcelona, Oikos-tau.

Vera, A. y RIVERA, J. (1999): Contribución invisible de las mujeres a la economía. El caso específico del mundo rural. Madrid: Ministerio de Asuntos Sociales, Instituto de la Mujer.

VILLARINo, M. y ARMAS, P. (2000): “La aldea global y el trabajo a domicilio en Galicia: coser y cantar», en Garcia Ramón, M. D. y Baylina Ferré, M.(eds.) (2000): Op. cit., (págs. 245-268). 